\title{
Motivos de adicción y riesgo de dependencia al tabaco en estudiantes de la licenciatura de enfermería
}

\author{
Gómez- Cardona Juan Pablo*, Ornelas-Picazo Francisco Javier**, De Lara Islas María de Jesús**, \\ Hernández-Macías Mariana Guadalupe**, Núnez-Díaz Nancy Carolina**, \\ Rodríguez- Nava Víctor Federico*, Rodríguez-Ramírez José Manuel*
}

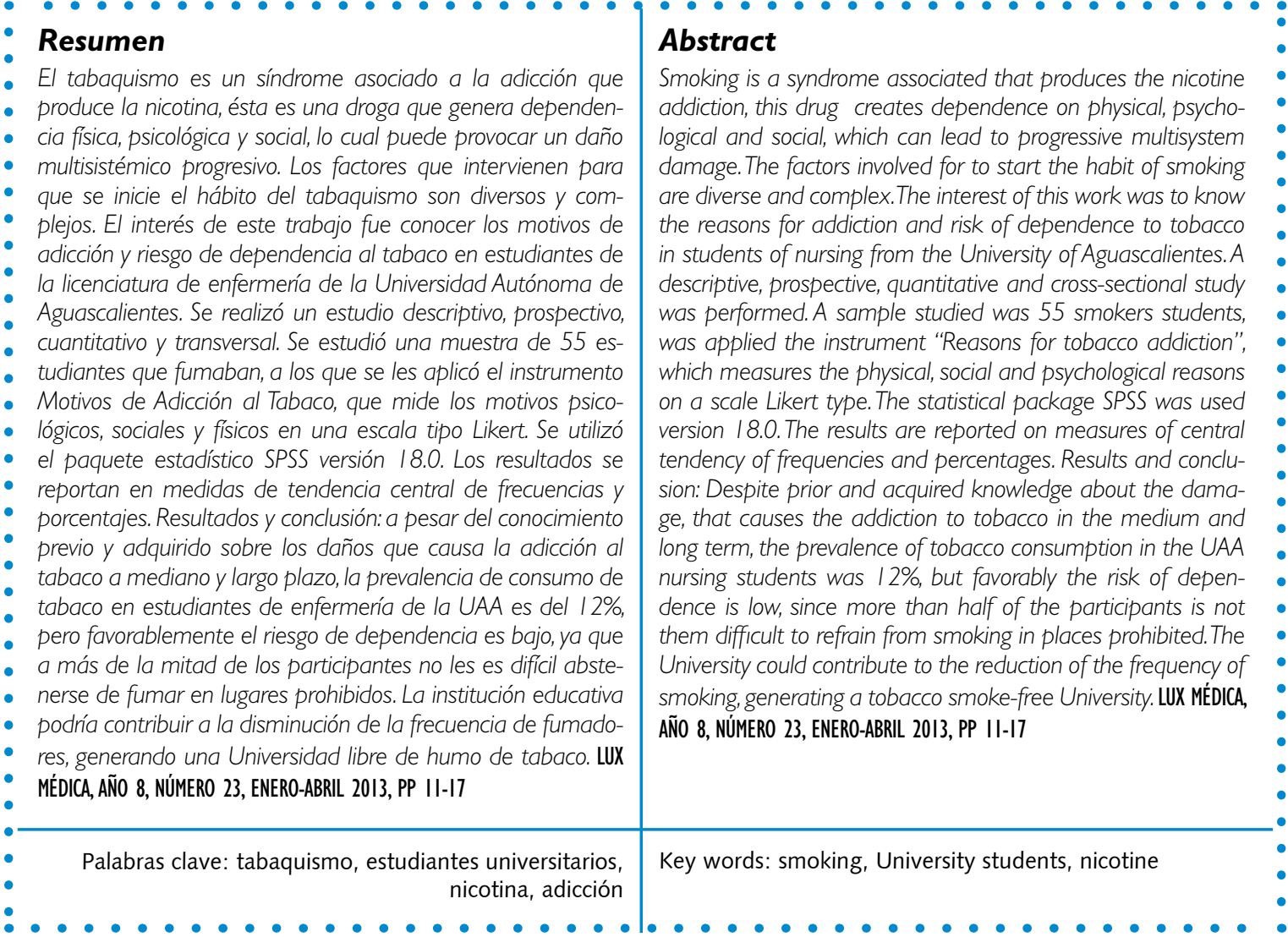

** Estudiantes de 8 semestre de Licenciatura en Enfermería de la Universidad Autónoma de Aguascalientes. Profesores de tiempo completo del Departamento de Enfermería del Centro de Ciencias de la Salud de la Universidad Autónoma de Aguascalientes

Fecha de recibido: 5 de marzo 2012

Fecha de aceptación: 15 de enero 2013

Correspondencia: MCE Juan Pablo Gómez Cardona. Departamento de Enfermería del Centro de Ciencias de la Salud de la Universidad Autónoma de Aguascalientes. Av. Universidad No 940 Edificio 105, Ciudad. Universitaria. CP 20131 Aguascalientes, Ags. México. Tel +52 (449) 9108437.

e-mail:jpgomezc@correo.uaa.mx 


\section{Introducción}

El tabaquismo es un síndrome asociado a la adicción que produce la nicotina. Ésta es una droga que genera dependencia física, psicológica y social, lo cual puede provocar un daño multisistémico progresivo, por tanto la Organización Mundial de la Salud (OMS) promueve en forma prioritaria que el personal del área de la salud evite fumar ya que éstos desempeñan una importante función como educadores en la promoción de comportamientos saludables en la población general. ${ }^{1}$

La Encuesta Nacional de Adicciones (ENA) y otros investigadores refieren que el inicio de consumo de tabaco es, en el $60 \%$ de los casos, por curiosidad. En un porcentaje menor, tanto hombres como mujeres han manifestado que el inicio del hábito del tabaquismo fue propiciado por la convivencia con familiares, amigos o compañeros fumadores (29.8\% y $26.9 \%$ respectivamente). ${ }^{2-4}$ Un fenómeno que se está observando, es que el consumo de cigarrillos en el sexo femenino se ha incrementado, y la edad de inicio se observa en rangos de edad más tempranos. ${ }^{5}$

Algunos estudios de conducta en estudiantes, explican cómo las influencias sociales intervienen en la decisión del estudiante para comenzar a fumar cigarrillos o no: uno de los motivos es el consumo experimental entre amigos. ${ }^{3}$ Mientras que otros han mostrado que la tensión nerviosa y la ansiedad afectan la tolerancia a la nicotina y la dependencia de ella. ${ }^{6}$

La dependencia al tabaco explica en forma importante su uso sostenido, generado en gran medida por el componente denominado nicotina. ${ }^{6}$ En México, una de las principales causas de adicción al tabaco es la curiosidad, comprendido dentro de los motivos psicológicos, asimismo un número importante de los casos está dentro de los factores sociales que se ven influenciados por la familia, amigos o compañeros fumadores. .,5,7-9 $^{2}$

Algunos estudios realizados en fumadores del área de la salud arrojan una prevalencia promedio del $14 \%$; la proporción es mayor en varones mencionando el factor psicológico como el principal motivo como inicio a la adicción, además es común en ambos géneros, que manifiesten que iniciaron a fumar en la etapa de la adolescencia. $6,10-12,14-16$

De acuerdo a los resultados del Examen Médico Automatizado (EMA) que realizó la Universidad Autónoma de Aguascalientes en el año 2011, refiere que el consumo de más de cuatro cigarrillos al día por estudiantes de enfermería es del $4.9 \% .{ }^{16}$

Las motivaciones del hábito tabáquico son diversos entre los intervienen los psicológicos, sociales y físicos que, en algún momento dado llevan al consumo regular y con ello a la dependencia. El interés de este estudio fue conocer los motivos de adicción y riesgo de dependencia al tabaco en estudiantes de Enfermería de la Universidad Autónoma de Aguascalientes. 


\section{Material y métodos}

Estudio cuantitativo, descriptivo, prospectivo y transversal. El universo lo constituyó 454 estudiantes de la Licenciatura en Enfermería de la Universidad Autónoma de Aguascalientes en el mes de septiembre de 2011, del cual se extrajo una muestra no probabilística, por conveniencia conformada por 55 sujetos que fumaban y que aceptaron participar, excluyendo a los que no tenían el hábito de fumar.

Se utilizó el instrumento Motivos de Adicción al Tabaco (MAT) extraído del proyecto Multiregional: Pros, Contras, Resilencia y Motivaciones: Un Modelo Predictivo para el Consumo de Alcohol y Tabaco, liderado por la Facultad de Enfermería de la Universidad Autónoma de Nuevo León, en coordinación con la Comisión Interamericana para el Control del Abuso de Drogas de la Organización de los Estados Americanos (OEA) y la Federación Mexicana de Facultades y Escuelas de Enfermería (FEMAFEE). El instrumento original está dirigido a bachilleres, por esa razón se realizó un estudio piloto en estudiantes de nivel superior (30 fumadores), a fin de darle fiabilidad en esta población, obteniendo 0,842 por alfa de Cronbach con un índice de confianza del $95 \%$, el instrumento mide los motivos psicológicos, sociales y físicos en una escala tipo Likert: nunca $=1$, rara vez $=2$, a veces $=3$, casi siempre $=4$ y siempre $=5$.

Se agregó el test Fagestrom para la medición del riesgo de dependencia contenido en el Catálogo Maestro de Guías de Práctica Clínica, SSA-108-08 con una escala de valoración de: 0 a 3 puntos dependencia baja, 4 a 7 puntos dependencia moderada, 8 puntos o más puntos: dependencia alta. Se utilizó el paquete estadístico SPSS versión 18.0 para un análisis descriptivo a través de las medidas de tendencia central, frecuencias y porcentajes.

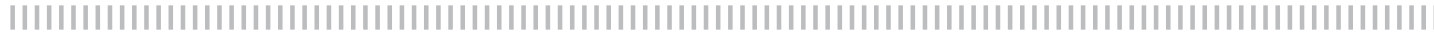

\section{Resultados}

El $12 \%$ de los estudiantes de enfermería refieren ser fumadores, de ellos el $60 \%$ corresponde al género femenino y el resto al masculino, las edades fluctuaron entre los 18 y 27 años, una media de 21 y una desviación estándar de 2.0 años. Los sujetos de estudio se ubican principalmente en los últimos semestres de la carrera y en el turno vespertino (Ver Tabla 1).

En la tabla 2, se aprecia que los principales motivos psicológicos para fumar son cuando los estudiantes se sienten frustrados, durante momentos de tensión, además de los estados de enojo, lo que hace suponer que se debe a la utilización del cigarrillo como un método de relajación con la finalidad disminuir el estrés. De los motivos sociales que principalmente sobresalen son el disfrutar prender el cigarro, por placer de aceptar y regalar cigarro, además de disfrutar desde el momento en que se agarra el cigarro. Los resultados obtenidos respecto a los motivos físicos, mencionan que nunca han sentido la necesidad de modificar sus hábitos referidos en las variables cuestionadas en el apartado, sin embargo llama la atención el incremento de porcentaje para el ítem en el que le atribuyen que a veces tienen problemas de salud (Ver Tabla 2)

De acuerdo con los resultados relacionados al nivel de riesgo de dependencia por el test Fagestrom, el $82 \%$ refiere fumar menos de 10 cigarrillos al día. El 55\% fuma más durante la primera parte del día. En cuanto al tiempo que transcurre desde que despierta hasta que fuma su primer cigarriIlo, el $70 \%$ lo hace posterior a la primera hora y el $30 \%$ entre los 30 y 60 minutos. El cigarro que más difícil es omitir es el primero de la mañana con el $89 \%$. En cuanto a dejar de fumar en lugares prohibidos el $55 \%$ expresa no serle difícil, mientras que 
al $45 \%$ se le dificulta. En relación a si fumar aun y cuando se encuentren enfermos, el $98 \%$ señala que puede prescindir de este hábito durante esta etapa. De acuerdo con lo anterior el test Fagestrom mostró que el riesgo de dependencia es baja a moderada principalmente en las mujeres, mientras que la dependencia alta está presente con mayor frecuencia en el género masculino (Ver Tabla 3).

\section{Tabla I}

\section{Distribución por sexo y semestre de los estudiantes fumadores de la carrera de Enfermería}

\begin{tabular}{|c|c|c|}
\hline Variable & $\%$ & $\mathbf{f}$ \\
\hline Fumadores & 12 & 55 \\
\hline No fumadores & 88 & 399 \\
\hline Total & 100 & 454 \\
\hline Género & $\%$ & $\mathbf{f}$ \\
\hline Mujeres fumadoras & 60 & 33 \\
\hline Hombres fumadores & 40 & 22 \\
\hline \multicolumn{3}{|l|}{$n=55$} \\
\hline Semestre que cursa & $\%$ & $\mathbf{f}$ \\
\hline Primero & 14.6 & 8 \\
\hline Segundo & 3.6 & 2 \\
\hline Tercero & 9.1 & 5 \\
\hline Cuarto & 3.6 & 2 \\
\hline Quinto & 9.1 & 5 \\
\hline Sexto & 16.4 & 9 \\
\hline Séptimo & 21.8 & 12 \\
\hline Octavo & 21.8 & 12 \\
\hline \multicolumn{3}{|l|}{$\mathrm{n}=55$} \\
\hline Turno escolar & $\%$ & $\mathbf{f}$ \\
\hline Matutino & 41.1 & 23 \\
\hline Vespertino & 58.2 & 32 \\
\hline $\mathrm{n}=55$ & & \\
\hline
\end{tabular}




\section{Tabla 2}

Motivos asociados al tabaquismo entre 55 estudiantes de enfermería de la UAA (sep 2011)

\begin{tabular}{|c|c|c|c|c|c|}
\hline $\begin{array}{l}\text { Psicológicos } \\
\text { Fumas cuando }\end{array}$ & $\begin{array}{c}\text { Nunca } \\
\%\end{array}$ & $\begin{array}{c}\text { Rara vez } \\
\% \\
\end{array}$ & $\begin{array}{c}\text { A veces } \\
\% \\
\end{array}$ & $\begin{array}{c}\text { Casi siempre } \\
\% \\
\end{array}$ & $\begin{array}{c}\text { Siempre } \\
\%\end{array}$ \\
\hline Te sientes frustrado. & 16,4 & 21,8 & 30,9 & 18,2 & 12,7 \\
\hline Te sientes enojado. & 27,3 & 30,9 & 25,5 & 12,7 & 3,6 \\
\hline Afrontas problemas. & 49,1 & 16,4 & 30,9 & 0 & 3,6 \\
\hline Estás tenso. & 10,9 & 23,6 & 40 & 14,5 & 10,9 \\
\hline Estás muy enojado con algo o alguien & 25,5 & 23,6 & 34,5 & 9,1 & 7,3 \\
\hline Te sientes triste o tienes preocupaciones & 29,1 & 27,3 & 25,5 & 10,9 & 7,3 \\
\hline $\begin{array}{l}\text { Sociales } \\
\text { Fumas porque }\end{array}$ & $\begin{array}{c}\text { Nunca } \\
\%\end{array}$ & $\begin{array}{c}\text { Rara vez } \\
\%\end{array}$ & $\begin{array}{c}\text { A veces } \\
\%\end{array}$ & $\begin{array}{c}\text { Casi siempre } \\
\% \\
\end{array}$ & $\begin{array}{c}\text { Siempre } \\
\%\end{array}$ \\
\hline Disfrutas al prender el cigarro & 29,1 & 30,9 & 30,9 & 3,6 & 5,5 \\
\hline Te sientes con más confianza con otra gente & 43,6 & 32,7 & 10,9 & 9,1 & 3,6 \\
\hline Te sientes más maduro y sofisticado. & 85,5 & 9,1 & 1,8 & 3,6 & 0 \\
\hline Da placer ofrecer y aceptar cigarros. & 58,2 & 10,9 & 23,6 & 7,3 & 0 \\
\hline Disfrutas desde que agarras el cigarro. & 47,3 & 16,4 & 20 & 5,5 & 10,9 \\
\hline Te sientes más atractivo. & 78,2 & 20 & 1,8 & 0 & 0 \\
\hline $\begin{array}{l}\text { Físicos } \\
\text { Fumas porque }\end{array}$ & $\begin{array}{c}\text { Nunca } \\
\%\end{array}$ & $\begin{array}{c}\text { Rara vez } \\
\%\end{array}$ & $\begin{array}{c}\text { A veces } \\
\%\end{array}$ & $\begin{array}{c}\text { Casi siempre } \\
\% \\
\end{array}$ & $\begin{array}{c}\text { Siempre } \\
\%\end{array}$ \\
\hline Has reducido o dejado el deporte & 67,3 & 7,3 & 10,9 & 7,3 & 7,3 \\
\hline Has dejado tus pasatiempos & 67,3 & 27,3 & 5,5 & 0 & 0 \\
\hline Sientes que eres adicto & 47,3 & 12,7 & 20 & 7,3 & 12,7 \\
\hline Has tenido problemas de salud & 41,8 & 23,6 & 27,3 & 3,6 & 3,6 \\
\hline Es difícil concentrarte cuando no traes cigarros. & 72,7 & 10,9 & 5,5 & 3,6 & 7,3 \\
\hline Es la primera cosa que haces por la mañana. & 70,9 & 9,1 & 9,1 & 3,6 & 7,3 \\
\hline
\end{tabular}

\section{Tabla 3}

Resultados del test de Fagerström sobre el riesgo de dependencia al tabaco según género en 55 estudiantes de la Lic. en Enfermería de la UAA (sep 2011)

\begin{tabular}{lccc} 
Motivos & $\begin{array}{c}\text { Mujeres } \\
\%\end{array}$ & $\begin{array}{c}\text { Hombres } \\
\%\end{array}$ & $\begin{array}{c}\text { Total } \\
\%\end{array}$ \\
\hline Dependencia baja & $33.5 \%$ & 22.3 & 55.8 \\
\hline Dependencia moderada & $17.4 \%$ & 13.4 & 30.8 \\
\hline Dependencia alta & $5.36 \%$ & 8.04 & 13.4 \\
\hline $\mathrm{n}=55$ & & & \\
\hline
\end{tabular}




\section{Discusión}

Coincidiendo con Pereyra ${ }^{7}$ y la ENA $^{1}$ las edades del grupo de estudio para estudiantes de nivel superior se encuentran entre los 18 a 20 años de edad y se ha suscitado un incremento del género femenino al hábito tabáquico, que se pudiera explicar por la cantidad de mujeres inscritas a una profesión tradicionalmente ejercida por ellas.

A pesar del fomento para que el personal médico y paramédico deje de fumar, ya que ellos desempeñan una importante función como educadores en la promoción de comportamientos saludables en la población general, existen evidencias que muestran que el profesional de la salud que tiene el hábito de fumar, no ofrecerá orientación a los usuarios sobre los riesgos del tabaquismo, ni se percibe como modelo, ${ }^{1,7}$ por lo que el presente estudio demuestra que el $12 \%$ de la población estudiantil de enfermería son fumadores, hasta un $14.6 \%$ durante el primer semestre probablemente asociado a los motivos sociales y generados durante su permanencia en secundaria y bachillerato, posteriormente disminuye el consumo en los periodos intermedios e incrementa nuevamente en los semestres finales que tal vez podría ser generadas por motivos psicológicos, $3,6,7,10,13-15$ tal como lo demuestra el presente estudio donde este motivo influye de manera importante destacando las variables: estar tenso, enojado con algo o alguien y frustrado.

Nuño y cols. así como Suárez y cols. mencionan que el principal motivo social es el consumo experimental entre amigos, ${ }^{4,5}$ mientras que el grupo de estudio refirió principalmente: disfrutar prender el cigarro, por placer de aceptar y regalar cigarro además de disfrutar desde el momento en que se agarra el cigarro, impulsos que se podrían creer superados por los estudiantes de nivel superior.

El EMA ${ }^{16}$ refiere que el $4.9 \%$ de los estudiantes de enfermería de primer semestre fuman 4 o más cigarrillos al día, mientras que el resultado de la encuesta es del $14.6 \%$, la diferencia puede radicar en la variable del test Fageström la opción es fumar 10 ó menos cigarrillos.

Coincidiendo con González ${ }^{2}$ la frecuencia del riesgo de dependencia al tabaco en ambas poblaciones corresponde a fumadores con riesgo bajo, por lo que es factible que abandonen el hábito con la concienciación sobre los daños que ocasiona y la restricción de las áreas para fumar.

\section{Conclusiones}

A pesar del conocimiento previo y adquirido sobre los daños que causa la adicción al tabaco a mediano y largo plazo; la prevalencia de consumo tabáquico en estudiantes de Enfermería de la UAA es del $12 \%$, pero favorablemente el riesgo de dependencia es bajo, aunado a que a más de la mitad no le es difícil abstenerse en lugares prohibidos; la institución educativa podría contribuir generando una Universidad libre de humo de tabaco. 


\section{Bibliografía}

1. México, Comisión Nacional contra las Adicciones. Encuesta Nacional de Adicciones 2008. Disponible en: (http://www.conadic.salud.gob.mx/pdfs/ena08/ ENA08_NACIONAL.pdf) consultado en Septiembre de 2011

2. González Enríquez L. Berger Vila K. Consumo de tabaco en adolescentes: factores de riesgo y factores protectores. Ciencia en Enfermería Chile 2002;8: disponible en: (http://www. scielo.cl/scielo.php?script=sci_arttext\&pid $=$ S071795532002000200004) Consultada en septiembre 2011.

3. Oropeza Abúndez C, Nagore Cázares G, Voghel Gutiérrez S, Rivero Vázquez S, Montiel Martínez P, Cortés González RA. Encuesta Nacional de Adicciones 2008. Instituto Nacional de Salud Pública. México 2009. 1era Edición. PP. 23-30.

4. Nuño Gutiérrez B.L. Álvarez Nemegyei J, Madrigal De León E.A. Tapia Curiel A. Factores asociados a los patrones de consumo de tabaco en adolescentes escolares. Revista Medica Instituto Mexicano Seguro Social. 2008; 46 (1): 19-26

5. Suarez Lugo N, Caraballoso Hernández M, Hechavarria Negrin S. El tabaquismo en los profesionales de la salud en el municipio Habana Vieja. Revista Cubana de Salud Publica, Ciudad de la Habana 2008. 34: disponible en http://scielo.sld.cu/scielo. php?pid =S0864-34662008000400002\&script $=$ sci arttext Consultada en septiembre de 2011.

6. Clemente-Jiménez $M$, Rubio-Aranda E, Perez-Trullén A, Marrón-Tundidor R, Herrero-Labarga I, Fernandez-Espinar J. Determinación de la dependencia a la nicotina en escolares fumadores a través del test de Fagerström modificado. An pediatr 2003; 58 (6) : 538-44.

7. Pereyra Martín D. S. Pereyra M. E. Tesis Prevalencia del consumo de tabaco en estudiantes de un campus universitario. Universidad Veracruzana. Minatitlán, ver 2008. Pp 1-35. disponible en: (http://cdigital. uv.mx/bitstream/12345678/958/1/tesis-0257.pdf) Consultado en Septiembre 2011.

8. Aguilar Rivera MM. Tesis prevalencia del consumo de tabaco en estudiantes de ciencias de la salud. Universidad Veracruzana. Minatitlán, Ver 2006. Pp 1-34. disponible en:(http://cdigital.uv.mx/bitstream/12345678/955/1/tesis-0253.pdf) consultada en septiembre 2011.
9. Arenas Monreal L, Jasso Victoria R, Martínez PC, Menjivar Rubio A. Prevalencia de tabaquismo de médicos y enfermeras en los estados de Morelos y Guanajuato. Revista del Instituto Nacional de Enfermedades Respiratorias 2004. 17: 261-265.

10. Pericas Beltrán J, Bennasar Veny M, González Torrente S, Seguí González P, De Pedro Gómez JE. Tabaquismo en enfermeras de Atención Primaria: Factores que influyen en su inicio y mantenimiento. Índex de Enfermería, Granada 2009. 18: disponible en: http://scielo.isciii.es/scielo.php?script=sci_ arttext\&pid =S1132-12962009000100005\&lng = es\&nrm =iso\&tlng $=$ es Consultada en septiembre 2011.

11. Ocampo Ocampo AM, Cuevas Aguirre E, Borjas Rivera I, Ramírez Casanova ME, Cicero Sabido R. ¿Quiénes fuman en un hospital general? Comentario clínico. Gaceta Médica de México 2001. 137:616620.

12. Llave Gamero Francisco Consumo de tabaco en adolescentes escolarizados de Almería disponible en: www.medynet.com/elmedico/publicaciones/.../512-515.pdf consultada en septiembre 2011.

13. Soto Masa F, Villalbí JR, Balcázar H, Valderrama Alberola J. La iniciación al tabaquismo: aportaciones de la epidemiología, el laboratorio y las ciencias del comportamiento. An Esp Pediatr 2002. 57:327-33

14. Arillo Santillán $E$, Thrasher J, Rodríguez Bolaños R, Chávez Ayala R, Ruiz Velasco S, Lazcano Ponce E. Susceptibilidad al consumo del tabaco en estudiantes no fumadores de 10 ciudades mexicanas. Salud Pública en México 2007. 49:170-181.

15. Rodríguez Gázquez MA, Pineda Botero SA, Vélez Yépes LF. Características del consumo de tabaco en estudiantes de enfermería de la Universidad de Antioquia Colombia. Investigación y Educación en Enfermería 2010. 28: 370-383.

16. México, Examen Médico Automatizado 2011, Caso Enfermería, Universidad Autónoma de Aguascalientes-Universidad Nacional Autónoma de México.

17. Chávez Domínguez RC, López Antuñano FJ, Regalado Pineda J, Espinosa Martínez M. Consumo de tabaco, una enfermedad social. Revista del Instituto Nacional de Enfermedades Respiratorias. 2004. 17:204-214. 


\section{Anexo 1}

\section{Instrumento MOTIVOS DE ADICCION AL TABACO}

\section{Datos generales}

\begin{tabular}{|l|l|l|}
\hline Sexo & Edad & Estado Civil \\
\hline Trabajas & Semestre & Turno \\
\hline
\end{tabular}

\section{Motivos psicológicos}

1.- ¿Te dan ganas de fumar cuando te sientes frustrado?

Nunca raravez a veces casi siempre siempre

2.- ¿Fumas cuando te sientes enojado?

Nunca rara vez a veces casi siempre siempre

3.- ¿Fumar te ayuda a afrontar los problemas o estresores de tu vida?

Nunca rara vez a veces casi siempre siempre

4.- ¿Fumar te ayuda cuando estás tenso?

Nunca rara vez a veces casi siempre siempre

5.- ¿Te dan ganas de fumar cuando estás muy enojado acerca de algo o con alguien?
Nunca
rara vez
a veces
casi siempre
siempre

6.- ¿Cuando te sientes triste o quieres poner tu mente en blanco acerca de preocupaciones, fumas cigarro?
Nunca
rara vez
a veces
casi siempre
siempre

\section{Motivos sociales}

7.- ¿Uno de los pasos que disfrutas es cuando prendes los cigarros?
Nunca
rara vez
a veces
casi siempre
siempre

8.- ¿Te sientes con más confianza con otra gente cuando estas fumando?
Nunca
rara vez
a veces
casi siempre
siempre

9. ¿Te sientes más maduro y sofisticado cuando estas fumando?
Nunca
rara vez
a veces
casi siempre
siempre

10.- ¿Fumas por el placer de ofrecer y aceptar cigarros de otra gente?
Nunca
rara vez
a veces
casi siempre
siempre

11.- ¿Al fumar un cigarro d disfrutas desde que tomos (agarras) el cigarro?

Nunca rara vez a veces casi siempre siempre

12.- ¿Te sientes más atractivo con el sexo opuesto cuando estas fumando?
Nunca
rara vez
a veces
casi siempre
siempre

\section{Motivos físicos}

13.- ¿Has reducido o dejado el ejerció o el deporte por fumar?
Nunca
rara vez
a veces
casi siempre
siempre

14.- ¿Has reducido a dejado pasatiempos cuando estas fumando?
Nunca
rara vez
a veces
casi siempre
siempre

15.- ¿Sientes que eres adicto a fumar cigarro?
Nunca
rara vez
a veces
casi siempre
siempre

16.- ¿Cres que has tenido algunos problemas de salud debido a fumar?
Nunca
rara vez
a veces
casi siempre
siempre

17. ¿Si vas sin cigarros por cierto tiempo encuentro difícil concentrarte?
Nunca
rara vez
a veces
casi siempre
siempre

18.- ¿Fumar un cigarro es la primer cosa que haces en la mañana?
Nunca
rara vez
a veces
casi siempre
siempre 


\section{Test de Fagerström}

\section{Riesgo de dependencia}

\section{1 ¿Cuántos cigarros fuma usted al día?}

\begin{tabular}{ll}
31 ó más & 3 puntos \\
\hline 21 a 30 & 2 puntos \\
\hline 11 a 20 & 1 punto \\
\hline Menos de 10 & 0 puntos \\
\hline
\end{tabular}

\section{2 ¿Fuma usted más cigarros durante la primera parte del día, que durante el resto del día?}

\begin{tabular}{ll} 
Sí & 1 punto \\
\hline No & 0 puntos \\
\hline
\end{tabular}

\section{3 ¿Cuánto tiempo transcurre desde que usted despierta hasta que fuma el primer cigarro?}

\begin{tabular}{lc}
3 puntos & Menos de cinco minutos \\
\hline 2 puntos & Seis a 30 minutos \\
\hline 1 punto & 31 a 60 minutos \\
\hline 0 puntos & Más de 60 minutos \\
\hline Conversión: & \\
\hline Una pipa = tres cigarros & \\
\hline Un puro = cuatro a cinco cigarros & \\
\hline Un puro pequeño = tres cigarros &
\end{tabular}

\section{4 ¿Qué cigarro le es más difícil de omitir?}

\begin{tabular}{lc} 
El primero de la mañana & 1 punto \\
\hline Algún otro & 0 puntos \\
\hline
\end{tabular}

\section{5 ¿Le es difícil no fumar donde ello es prohibido?}

\begin{tabular}{lc}
1 punto & Sí \\
\hline 0 puntos & No \\
\hline
\end{tabular}

\section{6 ¿Fuma usted cuando se halla enfermo e incluso en la cama?}

\begin{tabular}{ll} 
Sí & 1 punto \\
\hline No & 0 puntos \\
\hline
\end{tabular}

\section{Resultado de la prueba:}

De 0 a 3 puntos: Dependencia baja

De 4 a 7 puntos: Dependencia moderada

8 puntos o más puntos: Dependencia alta 